\title{
OBSTRUÇÃO INTESTINAL POR TRICOBEZOAR EM CÃO - RELATO DE CASO
}

Igor Henrique Vieira1, Marília de Oliveira Martins², Naílla Crystine de Carvalho Dias ${ }^{3}$, Carla Cristina Braz Louly ${ }^{4}$, Gabriella Canedo Costa ${ }^{5}$

1. Graduando em Medicina Veterinária da Universidade Federal de Goiás, Goiânia, Goiás, Brasil (igor_hv@hotmail.com);

2. Médica Veterinária autônoma;

3. Graduanda em Medicina Veterinária do Instituto Federal de Educação Ciência e Tecnologia Goiano - Campus Urutaí;

4. Professora Doutora do Departamento de Medicina Veterinária do Instituto

Federal de Educação Ciência e Tecnologia Goiano - Campus Urutaí;

5. Médica Veterinária autônoma.

Recebido em: 22/09/2018 - Aprovado em: 23/11/2018 - Publicado em: 03/12/2018 DOI: 10.18677/EnciBio_2018B62

\begin{abstract}
RESUMO
Tricobezoares são considerados concreções gastrintestinais decorrentes da ingestão de pelos. A ocorrência em cães é considerada incomum e tem sido associada a alterações de comportamento como a alotriofagia, estenose do trato gastrintestinal devido a tumor e por complicações cirúrgicas. Este relato objetiva descrever uma apresentação clínica incomum de um cão com obstrução do intestino delgado por um tricobezoar. Cão, fêmea, sem raça definida, nove anos de idade, foi atendido em uma clínica veterinária na cidade de Goiânia - Goiás, com apresentação de prostração, aquesia e vômito com odor fétido e cor escura. Ao exame físico, constatou-se mucosas pálidas, TPC maior que dois segundos, grau de desidratação moderada (8\%), distensão abdominal e massa com consistência rígida em região mesogástrica. Os resultados das análises bioquímicas apontaram diminuição na concentração sérica de albumina. O exame radiográfico evidenciou estômago e segmentos do intestino delgado acentuadamente distendidos por conteúdo gasoso e por conteúdo radiopaco de aspecto homogêneo. Os achados ultrassonográficos demonstraram íleo distendido por conteúdo hiperecóico associado à formação de sombra acústica. Em decorrência da gravidade do quadro, optou-se pela intervenção cirúrgica. Procedeu-se a enterectomia da região em que o tricobezoar estava alojado, devido a inviabilidade e necrose dessa porção do intestino. A paciente permaneceu internada por três dias após o procedimento cirúrgico, o animal apresentou vários episódios de hipotermia e hipotensão e piora do quadro clínico e veio a óbito.
\end{abstract}

PALAVRAS-CHAVE: cão, comportamento, bezoar.

\section{INTESTINAL OBSTRUCTION BY TRICOBEZOAR IN DOG - CASE REPORT}

ABSTRACT
Trichobezoars are considered gastrointestinal concretions resulting from the
ingestion of hairs. The occurrence is unusual in dogs and it is associated with 
abnormal behavioral like allotriophagy, stenosis of the gastrointestinal tract due to a tumor and surgical complications. This case report aims to describe an unusual clinical presentation of a dog with obstruction of the small intestine by a tricobezoar. Dog, female, without defined breed, nine years of age, was answered in a veterinary clinic in the city of Goiânia - Goiás, with presentation of prostration, aquesia and vomiting with a fetid odor and dark color. The physical examination revealed pale mucous membranes, TPC bigger than two seconds, degree of dehydration moderate $(8 \%)$, abdominal distension and mass with rigid consistency on mesogastric region. The results of biochemical analysis showed a decrease in serum concentration of albumin. The radiographic examination showed the stomach and segments of small intestine markedly distended by gaseous content and content of radiopaque homogeneous aspect. The ultrasonographic findings demonstrated lleum overdistended by hyperechogenic content associated with the formation of acoustic shadowing. Due to the severity of the condition, we opted for surgical intervention. Proceeded the enterectomy the region in which the Trichobezoar was housed, due to inviability and necrosis of this portion of the small intestine. The patient remained hospitalized for three days after the surgical procedure, the animal presented several episodes of hypothermia and hypotension and worsening of clinical and came to death.

KEYWORDS: dog, behavior, bezoar.

\section{INTRODUÇÃO}

Bezoares são considerados concreções de substâncias estranhas orgânicas ou inorgânicas, ingeridas voluntariamente, que se acumulam no trato gastrointestinal ou geniturinário (IWAMURO et al., 2015; JAIN et al., 2018). São classificados de acordo com o material que os forma, tais como, fitobezoares: conteúdo indigesto de frutas ou vegetais, tricobezoares: cabelo, lactobezoares: produtos lácteos e farmacobezoares: comprimidos e medicamentos (NOUR et al., 2017; SHAHRAZAD AKBAR et al., 2018), há também relatos de bezoares formados por fungos, Couceiro et al. (2016) e podem ser compostos ainda por plástico e até pedras (DIAS et al., 2010).

Os tricobezoares em humanos ocorrem frequentemente em pacientes jovens que sofrem de tricotilomania (compulsão em puxar o cabelo) e tricofagia (compulsão em engolir o cabelo) (CANNALIRE et al., 2018), sendo relatados acometendo o estômago, Lopez et al. (2014); Couceiro et al. (2016); Shahrazad Akbar et al. (2018) e sob a forma de "síndrome de Rapunzel" (OCHOA et al., 2018; PLASKETT et al., 2018; PRASAD et al., 2018), caracterizada pela presença de tricobezoar, estendendo-se do estômago para o intestino delgado (CANNALIRE et al., 2018).

$\mathrm{Na}$ Medicina Veterinária, a ocorrência do tricobezoar está associada a animais de pelos longos (DIAS et al., 2010), como coelhos (FERREIRA et al., 2007) e principalmente, felinos (DUROCHER et al., 2009; HAYNES et al., 2010), este último, resultado da rotina diária de higiene, durante a qual os animais lambem e ingerem o próprio pelo (LOUREIRO et al., 2014). Em cães, embora a obstrução intestinal secundária à ingestão de um corpo estranho, seja frequentemente observada, a obstrução intraluminal causada por um tricobezoar, é considerada incomum (ALBERNAZ et., al 2017) e já foi associada a alotriofagia (DIAS et al., 2010), estenose devido a tumor (O'Brien; Wong, 2001) e decorrente de complicações cirúrgicas, de anastomose final (CAROBBI et al. 2009) e sutura intestinal (ALBERNAZ et al., 2017). 
O diagnóstico é feito usando estudos radiológicos, como radiografia abdominal, com ou sem contraste e tomografia computadorizada ou ultrassonografia, que revelam estruturas calcificadas, granulares ou em redemoinhos de material sólido e gasoso (SHAHRAZAD AKBAR et al., 2018). A fragmentação endoscópica, tem sido frequentemente aplicada para bezoares gástricos. Uma vez feito o diagnóstico, o bezoar é geralmente dissolvido ou removido, porque pode causar obstrução, ulcerações devido à necrose por pressão, e subsequente sangramento gastrointestinal (IWAMURO et al., 2015).

A terapia deve ser adaptada à composição da concreção e ao processo fisiopatológico subjacente (COUCEIRO et al., 2016). Os tricobezoares são resistentes à degradação enzimática e à farmacoterapia (IWAMURO et al., 2015) e portanto, são retirados por meio de procedimentos cirúrgicos. Em razão dos escassos relatos desta enfermidade na espécie canina, este trabalho tem por objetivo descrever uma apresentação clínica incomum de um cão com obstrução do intestino delgado por um tricobezoar.

\section{RELATO DE CASO}

Cão, fêmea, sem raça definida, nove anos de idade, $4,6 \mathrm{~kg}$, foi atendido em uma clínica veterinária na cidade de Goiânia - Goiás, com apresentação de prostração, aquesia e vômito com odor fétido e cor escura. Foi relatado pelo tutor que o animal estava apático há aproximadamente sete dias apresentando episódios de vômito, hipoquesia e dor em região abdominal. Há cerca de três dias, começou a apresentar inapetência, alimentando-se de forma forçada com comida caseira. Segundo o tutor, em períodos em que a cadela permanecia sozinha em casa, tinha o hábito de consumir lixo, objetos e materiais que encontrasse. A desverminação e vacinas estavam atualizadas.

Ao exame físico, constatou-se mucosas pálidas, TPC maior que dois segundos, grau de desidratação moderada (8\%), frequência cardíaca de $130 \mathrm{bpm}$ e frequência respiratória de $31 \mathrm{mrpm}$. Nenhuma alteração foi identificada à auscultação cardiopulmonar. Os linfonodos palpáveis não apresentaram anormalidades. À palpação abdominal, notou-se distensão abdominal e massa com consistência rígida em região mesogástrica em antímero direito. Foram coletadas amostras sanguíneas para realização de hemograma, bioquímicas séricas (creatinina, ureia, alanina aminotransferase - ALT e albumina) e teste de glicemia capilar, além da realização de radiografia e ultrassonografia abdominais.

O hemograma não apresentou alterações. Os resultados das análises bioquímicas apontaram diminuição na concentração sérica de albumina: 1,73 g/dL (2,6 - 3,3 g/dL), enquanto que a ALT: $78 \mathrm{U} / \mathrm{L}(21-102 \mathrm{U} / \mathrm{L})$, creatinina: $0,9 \mathrm{mg} / \mathrm{dL}$ ( $0,5-1,5 \mathrm{mg} / \mathrm{dL}$ ) e a glicemia: $85 \mathrm{mg} / \mathrm{dL}(65-118 \mathrm{mg} / \mathrm{dL})$ permaneceram dentro dos valores de referência para a espécie. No exame radiográfico, foram observados estômago e segmentos de intestino delgado acentuadamente distendidos por conteúdo gasoso (alça de alerta) e segmento de intestino delgado acentuadamente distendido por conteúdo radiopaco de aspecto homogêneo, melhor visualizado em projeção lateral (Figura 1A), sendo os achados compatíveis com obstrução intestinal. Os achados ultrassonográficos, revelaram segmentos do intestino delgado (duodeno e jejuno) distendidos por conteúdo gasoso e líquido, e o íleo distendido por conteúdo hiperecóico, associado a formação de sombra acústica, também compatíveis com obstrução intestinal (Figura 1B). Não foram encontrados nos exames de imagem, alterações em outros órgãos. 


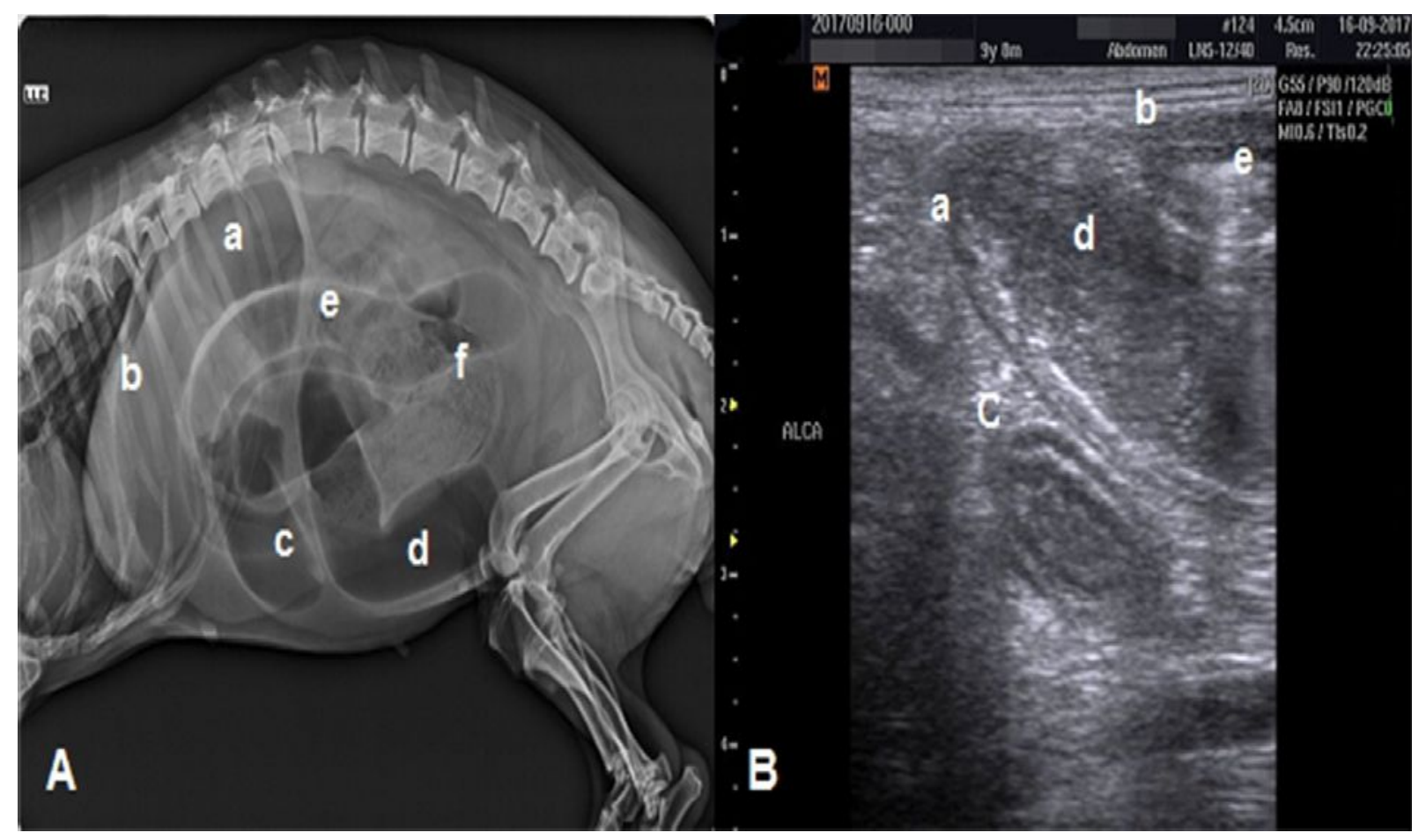

FIGURA 1 - Radiografia (A) e ultrassonografia (B) realizadas em paciente da espécie canina, fêmea, sem raça definida, com nove anos de idade, diagnosticada com obstrução intestinal. A) Estômago (a) e segmentos de intestino delgado acentuadamente distendidos por conteúdo radioluscente (alças de alerta) (b,c,d,e) e segmento de intestino delgado acentuadamente distendido por conteúdo radiopaco de aspecto homogêneo (f). B) Segmentos do intestino delgado (a, b, c) distendidos por conteúdo hipoecóico (líquido) (d) e hiperecóico (gasoso) (e). Fonte: Acervo pessoal.

Em decorrência da gravidade do quadro e da necessidade de tratamento cirúrgico, a paciente foi encaminhada para cirurgia. A antibioticoterapia profilática foi realizada por meio de administração de ceftriaxona dissódica $(30 \mathrm{mg} / \mathrm{kg}$, Amplospec ${ }^{\oplus}$, Biochimico, Rio de Janeiro, RJ). Como protocolo anestésico, utilizou-se a metadona $(0,2 \mathrm{mg} / \mathrm{kg}$, Cristália, Itapira, SP) como medicação pré-anestésica, e a indução foi realizada com propofol $(4 \mathrm{mg} / \mathrm{kg}$, Provive $\AA 1 \%$, Claris Produtos Farmacêuticos, Barueri, SP) e lidocaína ( $2 \mathrm{mg} / \mathrm{kg}$, Hypofarma, Ribeirão das Neves, $M G$ ) em bolus. Procedeu-se então, com a tricotomia e antissepsia prévia e definitiva. A paciente foi submetida à intubação orotraqueal, e a manutenção anestésica foi realizada por meio de isoflurano (Biochimico, Rio de Janeiro, RJ) diluído em oxigênio a $100 \%$.

O animal foi posicionado em decúbito dorsal em uma calha de inox, sobre colchão térmico, procedeu-se à celiotomia mediana pré retro-umbilical. $\mathrm{Na}$ exploração da cavidade abdominal, por meio de observação e palpação, detectou-se que a obstrução estava presente no lúmen do íleo. Este segmento foi exteriorizado e isolado sobre compressas cirúrgicas (Figura 2A). Após a enterotomia, efetuou-se a retirada de um corpo estranho composto por conteúdo fecal e cabelos, sendo identificado, portanto, como tricobezoar (Figura 2B). Efetuou-se a ligadura dos vasos 
mesentéricos e a enterectomia da região em que o tricobezoar estava alojado, devido a inviabilidade e necrose dessa porção do intestino.

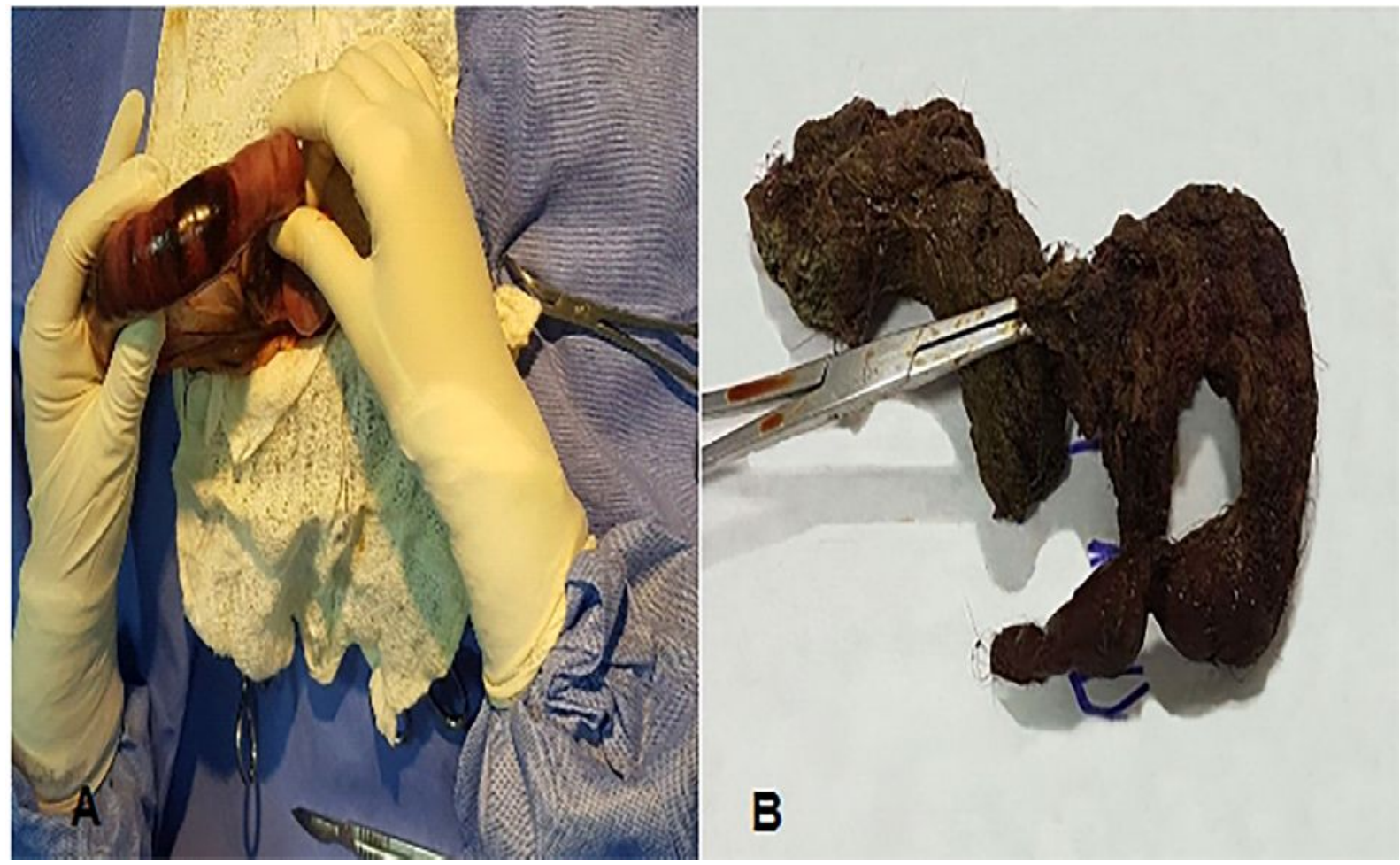

FIGURA 2 - Enterectomia para retirada de corpo estranho em paciente canina, fêmea, de nove anos de idade e tricobezoar. A) Exposição de segmento de intestino delgado (íleo) isolado sobre compressas cirúrgicas. B) Tricobezoar, medindo aproximadamente $15 \mathrm{~cm}$ de comprimento. Fonte: Acervo pessoal.

Após a limpeza das bordas da ferida cirúrgica com compressas de gaze estéril embebidas em solução fisiológica, efetuou-se a anastomose término terminal da alça com ponto simples separado, desde o defeito mesentérico, utilizando sutura absorvível monofilamentar poliglecaprone 3-0 (Poliglecaprone $25^{\circledR}$, Bioline, Anapólis, GO). Foi realizada a omentalização do segmento suturado, seguida de celiorrafia com nylon 2-0 (Shalon ${ }^{\circledR}$, Goiânia, GO) em padrão de sutura reverdin; a redução do subcutâneo foi realizada com ácido poliglicólico 2-0 (Shalon ${ }^{\circledR}$, Goiânia, GO), em padrão de sutura de cushing, por fim, foi feita a dermorrafia com nylon 3-0 (Nylon ${ }^{\circledR}$, Shalon, Goiânia, GO), em padrão de sutura de wolf.

A paciente permaneceu internada por três dias após a enterectomia, sendo submetida ao seguinte protocolo terapêutico: fluidoterapia com Ringer com Lactato $(5 \mathrm{ml} / \mathrm{kg})$ por hora, ceftriaxona dissódica $(30 \mathrm{mg} / \mathrm{kg}$, Amplospec®, Biochimico, Rio de Janeiro, RJ) a cada 12 horas, metronidazol (15 mg/kg, Endonidazol $0,5 \%{ }^{\circledR}$, Fresenius Kabi, Barueri, SP) a cada 12 horas, cloridrato de metoclopramida $(0,5$ $\mathrm{mg} / \mathrm{kg}$, Teuto, Anápolis, GO) a cada 8 horas, omeprazol (1 mg/kg, Teuto, Anápolis, GO) a cada 24 horas , citrato de maropitant (1 mg/kg, Pfizer, São Paulo, SP) a cada 24 horas, cloridrato de tramadol (4 mg/kg, Halexlstar, Goiânia, GO) a cada 8 horas e dipirona (25 mg/kg, Teuto, Anápolis, GO) a cada 8 horas.

Durante o pós-operatório imediato, foi colocada sonda nasogástrica para garantir a alimentação da paciente, já que a mesma apresentava anorexia. Foi realizada dieta zero nas primeiras 12 horas, sendo aspirado o conteúdo gástrico a 
cada hora. A partir desse período, procedeu-se com alimentação via sonda, feita pela administração de suplemento hipercalórico (5 $\mathrm{mL}$, Nutralife Intensive $\left.{ }^{\circledR}\right)$ preparado de acordo com instruções do fabricante e fornecido a cada três horas. Durante os três dias de internação, o animal apresentou vários episódios de hipotermia e hipotensão, sendo utilizados colchão térmico e infusão contínua de norepinefrina $(0,3 \mathrm{mcg} / \mathrm{kg}$, Cristália, Contagem, MG) por minuto, respectivamente, para controle da temperatura e hipotensão. No quarto dia de internação, a paciente sofreu uma parada cardiorrespiratória e foi a óbito.

\section{DISCUSSÃO}

Os tricobezoares são relativamente raros e importante causa de hiporexia e desnutrição em pequenos animais (DIAS et al., 2010). Uma das principais explicações para a ingestão de corpos estranhos por cães adultos, são os distúrbios comportamentais, como viver em confinamento, restrição de vida social e ausência de enriquecimento ambiental correto (NETO et al., 2011). Cães domésticos são diagnosticados para várias condições comportamentais relacionadas à ansiedade, tais como transtornos de ansiedade generalizada, fobias e ansiedade de separação, que em alguns casos podem ser considerados problemas graves de bem-estar (BLACKWELL et al., 2013). O tratamento consiste basicamente em modificação do ambiente, meio social e comportamento, e quando necessária intervenção farmacológica (DIAS et al., 2010).

No presente relato, após a exclusão de enfermidades sistêmicas por meio do exame clínico, exames de imagem e laboratoriais, a suspeita de causa desencadeante da ingestão de cabelo dos proprietários, se reafirma como uma alteração comportamental. De acordo com Gorter et al. (2010), o cabelo humano é resistente à digestão, bem como ao peristaltismo, devido à sua superfície lisa e leva a impactação, junto com muco e comida, causando a formação do tricobezoar.

Sinais clínicos de vômito intermitente, hipoquesia, diarreia e desidratação moderada foram observados em cães com tricobezoar gastrintestinal por O'brien e Wong (2001); Carobbi et al. (2009); Dias et al. (2010); Albernaz et al. (2017) e demonstrados no presente relato. De acordo com Hobday et al. (2014), os sintomas são inespecíficos e dependem de sua localização e o grau de obstrução. Ocasionalmente, o corpo estranho é palpado ao exame. O paciente pode às vezes, mostrar dor abdominal (NORDQUIST; CULP, 2013; HOBDAY et al., 2014), como observado no presente relato. No caso descrito, o tricobezoar encontrava-se exclusivamente no intestino delgado, alteração observada também por Carobbi et al. (2009); Dias et al. (2010) e Albernaz et al. (2017). Contrapondo estes relatos, Hettlich e Bahr (2000), demonstram um cão da raça Briard, de 12 anos de idade, com síndrome de Rapunzel.

Dentre as análises laboratoriais realizadas na paciente, observou-se a diminuição na concentração sérica de albumina; o hemograma e as outras bioquímicas não apresentaram alterações. Thrall et al. (2015) relatam dentre as causas de hipoalbuminemia em cães, a deficiência alimentar de fontes proteicas e o catabolismo aumentado da albumina, como consequência de deficiência energética; estes fatores poderiam justificar tal alteração na paciente deste relato, haja vista a hiporexia significativa apresentada pelo animal. Em humanos com tricobezoares gastrointestinais, a anemia já foi relatada por Lopez et al. (2014); Couceiro et al. (2016); Cannalire et al. (2018); Ochoa et al. (2018) e Shahrazad Akbar et al. (2018) e está relacionada a má absorção ou sangramento gastrointestinal (TABAAC, 2015). Em cães, a anemia foi observada no relato de Albernaz et al. (2017), o qual também 
se observou hipoalbuminemia, em um cão Poodle com obstrução intestinal por tricobezoar.

Bezoares gastrintestinais podem levar a complicações graves, incluindo obstrução do intestino delgado, isquemia intestinal ou perfuração (HIRJI et al., 2018). O animal relatado foi submetido à enterectomia, para resolução da obstrução intestinal e retirada do segmento do íleo que alojava o tricobezoar, já que apresentavam áreas de isquemia e necrose. Os procedimentos anestésicos e cirúrgicos foram realizados, segundo Fossum (2014). O protocolo de antibioticoterapia empregado no pós-operatório, baseou-se na associação de cefalosporina de terceira geração (ceftriaxona) com metronidazol. Esta associação é considerada eficiente, juntamente com uso de analgésico, no pós-operatório de cirurgias do sistema digestório (FOSSUM, 2014). O tratamento emergencial da hipotermia e da hipotensão seguiu o preconizado por Rabelo (2012).

Não foi possível saber ao certo, qual a complicação que ocasionou a morte da paciente, porém visto que choque, deiscência, perfuração, peritonite, estenose, síndrome do intestino curto, recidiva e morte são possíveis complicações da cirurgia intestinal (FOSSUM, 2014), e sendo o choque séptico, caracterizado por baixa da pressão arterial e temperatura corpórea, e pode ser causado por cirurgias complexas e sepse (RABELO, 2012), acredita-se que tenha sido esta, a complicação responsável pelo óbito.

\section{CONCLUSÃO}

Os tricobezoares na espécie canina, apesar de pouco relatados são observados na clínica de pequenos animais, e estão relacionados à enfermidades pré-existentes e alterações comportamentais. Portanto, a constatação da causa de base e o manejo comportamental e farmacológico adequado, devem ser instituídos pelo Médico Veterinário, a fim de solucionar o fator desencadeante e se evitar complicações.

\section{REFERÊNCIAS}

ALBERNAZ, V.G.P.; CONCEIÇÃO, R.T.; EISING, T.C.; FABRIS, I.A.; MAMPRIM, M.J. ET al. Partial Obstruction of the Small Intestine by a Trichobezoar in a Dog. Acta Scientiae Veterinariae, v. 45, n.1, 2017. Disponível em: http://www.ufrgs.br/actavet/45-suple-1/CR_210.pdf

BLACKWELL EJ, BRADSHAW JWS, CASEY RA. Fear responses to noises in domestic dogs: Prevalence, risk factors and co-occurrence with other fear related behaviour. Applied Animal Behavior Science, v.145, p. 15-25, 2013. Disponível em:<https://doi.org/10.1016/j.applanim.2012.12.004>.doi:10.1016/j.applanim.2012.12 .004

CANNALIRE, G.; CONTI, L.; CELONI, M.; GRASSI, C.; CELLA, A. et al. Rapunzel syndrome: an infrequent cause of severe iron deficiency anemia and abdominal pain presenting to the pediatric emergency department. BioMed Central Pediatrics, n. 18, p. 125, 2018. Disponível em: < https://doi.org/10.1186/s12887-018-1097-8>. doi: 10.1186/s12887-018-1097-8

CAROBBI, B.; FOALE, R. D.; WHITE, R.A.S. Trichobezoar Obstruction after Stapled Jejunal Anastomosis in a Dog. Veterinary Surgery, v. 38, p. 417- 420, 2009. 
Disponível em: < https://doi.org/10.1111/j.1532-950X.2008.00478.x>. doi:10.1111/j.1532-950X.2008.00478.x

COUCEIRO, A.; VIVEIRO, C.; CAPELÃO, G.; NOBRE, J.; LAUREANO, M. et al. Trichobezoar- A Rare Cause of Abdominal Mass and Gastric Outlet Obstruction - GE Portuguese Journal of Gastroenterology, v. 23, n. 1, p. 50- 53, 2016. Disponível em: < http://dx.doi.org/10.1016/j.jpge.2015.08.003>. doi: 10.1016/j.jpge.2015.08.003

DIAS, T. A.; FARIA, M. A. R.; ÁVILA, D. F.; CESARINO, M.; TANNÚS, L.F.; CASTRO, J. R. Tricobezoar gástrico decorrente de transtorno compulsivo em um cão Relato de caso. PUBVET, v. 4, n. 4, 2010. Disponível em: http://www.pubvet.com.br/uploads/2cba746edb70582ee1e8953b4ac42310.pdf

DUROCHER, L.; JOHNSON, S.E.; GREEN, E. Esophageal diverticulum associated with a trichobezoar in a cat. Journal of the American Animal Hospital Association, v. 45, n. 3, p. 142-146, 2009. Disponível em: < https://doi.org/10.5326/0450142>. doi:10.5326/0450142

FERREIRA, M. P.; ALIEVI, M. M.; BECK, C.A.C.; FILHO, A. P. F.S.; JUNIOR, E.B.S.; Corpo estranho gástrico em um coelho (Oryctolagus cuniculus). Acta Scientiae Veterinariae, v.35, n.2, p.249-251, 2007. Disponível em: https: //lume.ufrgs.br/handle/10183/20603

FOSSUM, T. W. Cirurgia de Pequenos Animais. 4 ed. Rio de Janeiro: Elsevier, 2014.

GORTER, R. R.; KNEEPKENS, C. M. F.; MATTENS, E. C. J. L.; ARONSON, D. C.; HEIJ, H. A.Management of trichobezoar: case report and literature review. Pediatric Surgery Internacional, v.26, p. 457-463, 2010. Disponível em: < https://doi.org/10.1007/s00383-010-2570-0>. doi: 10.1007/s00383-010-2570-0

HAYNES, K. J.; ANDERSON, S. E.; LASZLO, M. P. Nasopharyngeal trichobezoar foreign body in a cat . Journal of Feline Medicine and Surgery, v.12, p. 878 - 881, 2010. Disponível em: <https://doi.org/10.1016/j.jfms.2010.09.008>. doi:10.1016/j.fms.2010.09.008

HETTLICH, B.F.; BAHR, A.M. What is your diagnosis? Soft tissue opacity in stomach and duodenum. Journal of American Veterinary Medical Association, v. 217, n.4, p. 477- 478, 2000. Disponível em: < http://dx.doi.org/10.2460/javma.2000.217.477 >. doi: 10.2460/javma.2000.217.477

HIRJI, S.A.; ROBERTSON, F.C.; CHAO, G. F.; KHURANA, B.;GATES, J.D. Phytobezoar: A Brief Report with Surgical and Radiological Correlation. Case Reports in Surgery, 2018. Disponível em: https://doi.org/10.1155/2018/5253162>. doi: $10.1155 / 2018 / 5253162$

HOBDAY, M. M.; PACHTINGER,G.E.; DROBATZ,K.J.; SYRING, R.S. Linear versus non-linear gastrointestinal foreign bodies in 499 dogs: clinical presentation, management and short-term outcome. Journal of Small Animal Practice. v.55, 
p.560-565, 2014. Disponível em: < https://doi.org/10.1111/jsap.12271>. doi: $10.1111 /$ jsap.12271

IWAMURO, M.; OKADA, H.; MATSUEDA, K.; INABA, T.; KUSUMOTO, C. et al. Review of the diagnosis and management of gastrointestinal bezoars. World Journal Gastrointestinal Endoscopy, v.7, p. 336-345, 2015. Disponível em: <https://doi.org/10.4253/wjge.v7.i4.336>. doi: 10.4253/wjge.v7.i4.336

JAIN, S.A.; AGARWAL, L.; KHYALIA, A.; CHANDOLIA,P.; KAKNALE, H. Pharmacobezoar - a rare case presented as gastric outlet obstruction. Journal of surgical case reports, n.5, 2018. Disponível em:<https://doi.org/10.1093/jscr/rjy116>. doi: 10.1093/jscr/rjy116

LOPEZ, A.J.F.; VALVERDE, F.M.G.; MONCADA, J.R.; BLAZQUEZ, A. A. M. Trichophagia and Trichobezoar. Cirugía Española, v.92, n.6, p.448-449, 2014. Disponível em: < https://doi.org/10.1016/j.ciresp.2014.01.007>. doi:10.1016/j.ciresp.2014.01.007

LOUREIRO, B. A.; SEMBENELLI, G.; MARIA, A.P.J.; VASCONCELLOS, R.S.; SÁ, F.C. et.al. Sugarcane fibre may prevents hairball formation in cats. Journal of Nutritional Science, $\quad$ v.3, $2014 . \quad$ Disponível em: <https://doi.org/10.1017/jns.2014.27>. doi:10.1017/jns.2014.27

NETO, J. M. C.; CONCEIÇÃO, I. R.; SEIXAS, M. V.; TORÍBIO, J. M. M. L., et al. Alotriofagia -manifestação de transtorno obsessivo-compulsivo em um cão: relato de caso. Medicina Veterinária, v.5 n.3, p.27-32, 2011. Disponível em: http://journals.ufrpe.br/index.php/medicinaveterinaria/article/view/642/521

NORDQUIST, B.; CULP, W.T.N. Focal and Linear Gastrointestinal Obstruction.Small Animal Soft Tissue Surgery, p. 360-73, 2013. Disponível em://http://doi.org/10.1002/9781118997505.ch38>.doi:10.1002/9781118997505.ch38

NOUR, I.; ALATEF, M.A.; MEGAHED, A.;YAHIA, S.; WAHBA, Y.;SHABAAN, A.E. Rapunzel syndrome (gastric trichobezoar), a rare presentation with generalized edema: case report and review of the literature. Paediatrics and International Child Health, v. 23, p.1-3, 2017. Disponível em: < https://doi.org/10.1080/20469047.2017.1389809>.doi:10.1080/20469047.2017.138 9809

O'BRIEN, C.R.; WONG, W.T. Intermittent vomiting and weight loss in an old dog. Australian Veterinary Journal, v.79, n.4, p. 251-260, 2001. Disponível em: <https://doi.org/10.1111/j.1751-0813.2001.tb11975.x>. doi: 10.1111/j.17510813.2001.tb11975.x

OCHOA, M. B.; HERNÁNDEZ, M. X.; TORRES, M. E.; GAMBOA, A. Q.; LAVADORES, I. T. et al. Rapunzel syndrome with double simultaneous trichobezoar in a teenager: Clinical Case Reports, v.89, n.1, p.98-102, 2018. Disponível em: < https://doi.org/10.4067/S037041062018000100098>.doi:10.4067/S037041062018000100098. 
PLASKETT, J.; CHINNERY, G.; THOMSON, D.; THOMSON, S.; DEDEKIND, B. et al. Rapunzel syndrome: A South African variety. South African Medical Journal, v.108, n.7, p. 559-562, 2018. Disponível em:< https://doi.org//10.7196/SAMJ.2018.v108i7.13115>.doi:10.7196/SAMJ.2018.v108i7.1 3115.

PRASAD, A.; JAIN, A.; GUPTA, A.; KAMRA, A. Trichobezoar: Ravenous for Hair. Euroasian Journal Hepato-Gastroenterology, v. 8, n.1, p.97-98, 2018. Disponível em:<https://doi.org/10.5005/jp-journals-10018-1271>.doi:10.5005/jp-journals-100181271

RABELO, R. Sepse, Sepse Grave e Choque Séptico. In: Emergências em pequenos animais: Condutas clínicas e cirúrgicas no paciente grave. Rio de Janeiro, RJ: Ed. Elsevier, p. 322-340, 2012.

SHAHRAZAD AKBAR.; AKBAR. L.; AKBAR, N.; NAWRAS, A. Trichobezoar-Induced Heartburn in a Teenage Girl: A Case Report. Journal of Investigative Medicine High Impact Case Reports, v.6, p. 1-3, 2018. Disponível em: < https://doi.org/10.1177/2324709618776345>. doi: 10.1177/2324709618776345

TABAAC, B.J.; TABAAC, V. Pica patient, status post gastric bypass, improves with change in medication regimen. Therapeutic Advances in Psychopharmacology. v.5, n.1, p.38 - 42, $2015 . \quad$ Disponível em: <https://doi.org/10.1177/2045125314561221 > doi: 10.1177/2045125314561221.

THRALL, M.A.; WEISER, G.; ALLISON, R.W.; CAMPBELL, T.W. Avaliação Laboratorial das Proteínas do Plasma e do Soro Sanguíneo. In: Hematologia e Bioquímica Clínica Veterinária. 2ª edição. São Paulo: Roca, 2015, p. 398-412. 